\title{
Erratum
}

Fang-Hua Lin · Tristan Rivière

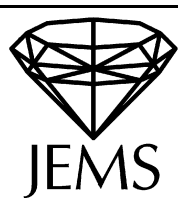

\section{Complex Ginzburg-Landau equations in high dimensions and codimension two area minimizing currents}

\author{
J. Eur. Math. Soc. 1, 237-311 (1999)
}

In our previous paper [2] "Complex Ginzburg-Landau equations in high dimensions and codimension two area minimizing currents", we studied the asymptotic behavior of energy minimizing solutions of the GinzburgLandau equations. But the $\eta$-compactness Lemma was for arbitrary solutions which may not be energy minimizing. We found there is a gap in this version of the proof of the $\eta$-compactness Lemma (Lemma II.7). This $\eta$-compactness Lemma as well as asymptotic behavior of arbitrary solutions are treated in our forthcoming paper [3]. Here we shall simply present our earlier proof of the $\eta$-compactness for energy minimizing solutions. All the statements in the rest of the paper [2] are not affected by this modification.

The arguments from (II.60) to (II.68) have to be modified in the following way. Starting from (II.60) we say:

In fact we will be mainly interested in $p$ such that $W^{1, p}\left(\partial B_{t}\right) \hookrightarrow H^{\frac{1}{2}}\left(\partial B_{t}\right)$, that is $p \geqslant 2-\frac{1}{n}$.

We will now extend the map $\tilde{u}$ into a map $w$ in all of $B_{t}$ by using as less energy as possible.

We will decompose $\partial B_{t}$ into a union of disjoint "cubes" having edges of length $\delta$, where $\delta$ will be taken very small (to be fixed later).

We will first extend $\tilde{u}$ between $\partial B_{t}$ and $\partial B_{t-\delta}$. As it is proved in [1] it is possible to choose this union of cubes such that, if $\mathcal{C}_{k}$ denotes the corresponding $k$-skeleton for this union of cubes (for $2 \leqslant k \leqslant n-1$ ), we

F.H. Lin: Courant Institute of Mathematical Sciences, New York University, 251 Mercer Street, New York, NY 10012-1185, USA

T. Rivière: Ecole Normale Supèrieure de Cachan, Centre de Mathèmatiques et de leurs Applications, Unitè associeè au CNRS URA-1611, 61, avenue du prèsident Wilson, F-94235 Cachan Cedex, France 
have in the same time

$$
\left\{\begin{array}{l}
\int_{\mathcal{C}_{k}}|\nabla \tilde{u}|^{2} \leqslant K\left(\frac{1}{\delta}\right)^{n-1-k} \int_{\partial B_{t}}|\nabla \tilde{u}|^{2} \quad \forall 2 \leqslant k \leqslant n-1 \\
\left(\frac{r_{1}}{\varepsilon}\right)^{2} \int_{\mathcal{C}_{k}}\left(1-|\tilde{u}|^{2}\right)^{2} \leqslant K\left(\frac{1}{\delta}\right)^{n-1-k}\left(\frac{r_{1}}{\varepsilon}\right)^{2} \int_{\partial B_{t}}\left(1-|\tilde{u}|^{2}\right)^{2} \\
\forall 2 \leqslant k \leqslant n-1 \\
\int_{\mathcal{C}_{k}}\left|\tilde{u} \wedge d_{\top} \tilde{u}\right|^{p} \leqslant K\left(\frac{1}{\delta}\right)^{n-1-k} \int_{\partial B_{t}}\left|\tilde{u} \wedge d_{\top} \tilde{u}\right|^{p} \\
\forall 2 \leqslant k \leqslant n-1 .
\end{array} .\right.
$$

Where $K$ is a constant depending only on $n$ not on $\varepsilon$ nor on $\delta$. First of all, $\delta$ will be chosen such that $|\tilde{u}| \geqslant 1 / 2$ in $\mathcal{C}_{2}$. Combining (II.47) of [2] and (1) we get

$$
\left\{\begin{array}{l}
\left(\frac{r_{1}}{\varepsilon}\right)^{2} \int_{\mathcal{C}_{2}}\left(1-|\tilde{u}|^{2}\right)^{2} \leqslant K\left(\frac{1}{\delta}\right)^{n-3} \eta \\
\|\nabla \tilde{u}\|^{2} \leqslant C \frac{r_{1}}{\varepsilon} .
\end{array}\right.
$$

Thus, this is the case if $\eta / \delta^{n-3}$ is sufficiently small (independantly of $\varepsilon$ ). Denote by $\mathcal{C}_{k}^{\delta}$ the $k$-skeleton homothetic to $\mathcal{C}_{k}$, contained in $\partial B_{t-\delta}$, for the homothetie of rate $\frac{t-\delta}{t}$ and let $\mathcal{D}_{k+1}$ be the $k+1$-skeleton in the interior of $B_{t} \backslash B_{t-\delta}$ having $\mathcal{C}_{k} \cup \mathcal{C}_{k}^{\delta}$ as boundary. we will construct the extension $w$ of $\tilde{u}$ in $B_{t} \backslash B_{t-\delta}$ on $\mathcal{D}_{k}$ by induction on $k$. For $k=3$ we take

$$
w(x)=\tilde{u}\left(t \frac{x}{|x|}\right) \quad \text { in } \mathcal{D}_{3} .
$$

We clearly have

$$
\left\{\begin{array}{l}
\int_{\mathcal{D}_{3}}|\nabla w|^{2} \leqslant \delta \int_{\mathcal{C}_{2}}|\nabla \tilde{u}|^{2} \leqslant K\left(\frac{1}{\delta}\right)^{n-4} \int_{\partial B_{t}}|\nabla \tilde{u}|^{2} \\
\left(\frac{r_{1}}{\varepsilon}\right)^{2} \int_{\mathcal{D}_{3}}\left(1-|w|^{2}\right)^{2} \leqslant K\left(\frac{1}{\delta}\right)^{n-4}\left(\frac{r_{1}}{\varepsilon}\right)^{2} \int_{\partial B_{t}}\left(1-|\tilde{u}|^{2}\right)^{2} \\
\int_{\mathcal{D}_{3}}\left|w \wedge d_{\top} w\right|^{p} \leqslant K\left(\frac{1}{\delta}\right)^{n-4} \int_{\partial B_{t}}\left|\tilde{u} \wedge d_{\top} \tilde{u}\right|^{p}
\end{array}\right.
$$


Before to construct the extension $w$ of $\tilde{u}$ in the interior of $B_{t} \backslash B_{t-\delta}$ we construct the extension $w$ of $\tilde{u}$ in $\partial B_{t}$, on the $\mathcal{C}_{k}^{\delta}$ by induction on $k$. On each cell of the 3 -skeleton $\mathcal{C}_{3}^{\delta}$, extend $w$ radially from the boundary of the cell, where $w(x)=\tilde{u}\left(t \frac{x}{|x|}\right)$, to the center of the cell. One verifies that we have

$$
\left\{\begin{array}{l}
\int_{\mathcal{C}_{3}^{\delta}}|\nabla w|^{2} \leqslant C \delta \int_{\mathcal{C}_{2}^{\delta}}|\nabla w|^{2} \leqslant C\left(\frac{1}{\delta}\right)^{n-4} \int_{\partial B_{t}}|\nabla \tilde{u}|^{2} \\
\left(\frac{r_{1}}{\varepsilon}\right)^{2} \int_{\mathcal{C}_{3}^{\delta}}\left(1-|w|^{2}\right)^{2} \leqslant C\left(\frac{1}{\delta}\right)^{n-4}\left(\frac{r_{1}}{\varepsilon}\right)^{2} \int_{\partial B_{t}}\left(1-|\tilde{u}|^{2}\right)^{2} \\
\int_{\mathcal{C}_{3}^{\delta}}\left|w \wedge d_{\top} w\right|^{p} \leqslant C\left(\frac{1}{\delta}\right)^{n-4} \int_{\partial B_{t}}\left|\tilde{u} \wedge d_{\top} \tilde{u}\right|^{p} \\
|w| \geqslant \frac{1}{2} \quad \text { a. e. in } \mathcal{C}_{3}^{\delta} .
\end{array}\right.
$$

Repeating these extensions on $\mathcal{C}_{k}^{\delta}$ until $k=n-1$ we obtain that

$$
\left\{\begin{array}{l}
\int_{\partial B_{t-\delta}}|\nabla w|^{2} \leqslant C \int_{\partial B_{t}}|\nabla \tilde{u}|^{2} \\
\left(\frac{r_{1}}{\varepsilon}\right)^{2} \int_{\partial B_{t-\delta}}\left(1-|w|^{2}\right)^{2} \leqslant C\left(\frac{r_{1}}{\varepsilon}\right)^{2} \int_{\partial B_{t}}\left(1-|\tilde{u}|^{2}\right)^{2} \\
\int_{\partial B_{t-\delta}}\left|w \wedge d_{\top} w\right|^{p} \leqslant C \int_{\partial B_{t}}\left|\tilde{u} \wedge d_{\top} \tilde{u}\right|^{p} \\
|w| \geqslant \frac{1}{2} \quad \text { a. e. in } \partial B_{t-\delta} .
\end{array}\right.
$$

Now we construct $w$ in $\mathcal{D}_{k}$ by induction on $k$. We have $\partial \mathcal{D}_{4}=\mathcal{D}_{3} \cup \mathcal{C}_{3} \cup \mathcal{C}_{3}^{\delta}$. Here also, in each cell, we choose $w$ to be the radial extension, relative to the center of the cell, from it's value on the boundary. We establish in the same way inequalities like (2), replacing $\mathcal{D}_{3}$ by $\mathcal{D}_{4}$ and $n-4$ by $n-5$. Repeating this procedure until $k=n$, we get in particular

$$
\left\{\begin{array}{l}
\int_{\mathcal{D}_{n}=B_{t} \backslash B_{t-\delta}}|\nabla w|^{2} \leqslant C \delta \int_{\partial B_{t}}|\nabla \tilde{u}|^{2} \\
\left(\frac{r_{1}}{\varepsilon}\right)^{2} \int_{B_{t} \backslash B_{t-\delta}}\left(1-|w|^{2}\right) \leqslant C \delta\left(\frac{r_{1}}{\varepsilon}\right)^{2} \int_{\partial B_{t}}\left(1-|\tilde{u}|^{2}\right)^{2} .
\end{array}\right.
$$


Finally we extend $w$ in $B_{t-\delta}$ in the following way. We have $|w| \geqslant 1 / 2$ in $\partial B_{t-\delta}$ a. e. and $w \in W^{1,2}\left(\partial B_{t-\delta}\right)$. Thus $\frac{w}{|w|} \wedge d \frac{w}{|w|} \in L^{2}\left(\partial B_{t-\delta}\right)$ and since

$$
d\left[\frac{w}{|w|} \wedge d \frac{w}{|w|}\right]=0
$$

and $H_{d R}^{1}\left(\partial B_{t-\delta}\right)=0$, there exists $\phi \in W^{1,2}\left(\partial B_{t-\delta}\right)$ such that

$$
\left\{\begin{array}{l}
\frac{w}{|w|} \wedge d \frac{w}{|w|}=d \phi \\
\text { and } \int_{\partial B_{t-\delta}} \phi=0 .
\end{array}\right.
$$

Thus $w=|w| \exp i \phi+i \phi_{0}$, where $\phi_{0} \in[0,2 \pi$ ), and from (II.68) of [2] and (3) we deduce that

$$
\|\phi\|_{W^{1, p}\left(\partial B_{t-\delta}\right)} \leqslant C_{q} \eta^{\frac{\gamma}{2}}\left(\int_{T_{1}}|\nabla \tilde{u}|^{2}\right)^{\frac{1}{2}}+C_{q} \eta^{\frac{\gamma}{2}}
$$

for some fixed $0<\gamma<1$, where $p=2-\frac{1}{n}$. By Sobolev embedding we have

$$
\|\phi\|_{H^{\frac{1}{2}\left(\partial B_{t-\delta}\right)}} \leqslant C \eta^{\frac{\gamma}{2}}\left(\int_{T_{1}}|\nabla \tilde{u}|^{2}\right)^{\frac{1}{2}}+C \eta^{\frac{\gamma}{2}} .
$$

Let $\bar{\phi}$ be the harmonic extension of $\phi$ in $B_{t-\delta}$, we have

$$
\int_{B_{t-\delta}}|\nabla \bar{\phi}|^{2} \leqslant C\|\phi\|_{H^{\frac{1}{2}\left(\partial B_{t-\delta}\right)}} \leqslant C \eta^{\gamma} \int_{T_{1}}|\nabla \tilde{u}|^{2}+C \eta^{\gamma}
$$

We take $w /|w|=\exp i \bar{\phi}+i \phi_{0}$ in $B_{t-\delta}$. For the modulus $|w|$ of $w$ in $B_{t-\delta}$ we choose $w=\omega$, where $\omega$ is the solution of the following problem

$$
\left\{\begin{array}{l}
-\left(\frac{\varepsilon}{r_{1}}\right) \Delta \omega+\omega=1 \quad \text { in } B_{t-\delta} \\
\omega=|w| \quad \text { in } \partial B_{t-\delta}
\end{array}\right.
$$

In [4] we proved that $\min _{B_{t-\delta}} \omega \geqslant \min _{\partial B_{t-\delta}}|w| \geqslant 1 / 2$ and

$$
\begin{aligned}
& \int_{B_{t-\delta}}|\nabla \omega|^{2}+\left(\frac{r_{1}}{\varepsilon}\right)^{2}\left(1-\omega^{2}\right)^{2} \\
& \leqslant C\left(\int_{\partial B_{t}}\left(1-|\tilde{u}|^{2}\right)^{2}\right)^{\frac{1}{2}} \times\left(\int_{\partial B_{t}}|\nabla \tilde{u}|^{2}+\left(\frac{r_{1}}{\varepsilon}\right)^{2}\left(1-|\tilde{u}|^{2}\right)^{2}\right)^{\frac{1}{2}}
\end{aligned}
$$


Combining (4), (5), (7) and the minimality of $\tilde{u}$ in $B_{t}$ we get

$$
\begin{aligned}
\int_{B_{t}}|\nabla \tilde{u}|^{2}+\left(\frac{r_{1}}{\varepsilon}\right)^{2}\left(1-|\tilde{u}|^{2}\right)^{2} & \leqslant C \eta^{\gamma} \int_{T_{1}}|\nabla \tilde{u}|^{2}+ \\
& +C \delta \int_{\partial B_{t}}|\nabla \tilde{u}|^{2}+C \eta^{\gamma} .
\end{aligned}
$$

Choose $\delta=C \eta^{\frac{1}{n-3}}$ (recall that we had already chosen $\left.\delta \geqslant C \eta^{\frac{1}{n-3}}\right)$.

Using (II.44) of [2] and going back to the usual scale we get, for $\beta=$ $\inf \left(\frac{1}{n-3}, \gamma\right)$,

$$
\frac{E_{t r_{1}}}{\left(t r_{1}\right)^{n-2}} \leqslant C \eta^{\beta} \frac{E_{r_{1}}}{r_{1}^{n-2}}+\eta^{\beta}
$$

where $t \geqslant 1 / 2$. Thus, because of the monotonicity formula we have (II.68) of [2] and the proof can be ended like in [2].

\section{References}

1. F. Bethuel: The approximation problem for Sobolev maps between two manifolds. Acta Math. 167, 153-206 (1991)

2. F.H. Lin, T. Rivière: Complex Ginzburg-Landau equations in high dimensions and codimension two area minimizing currents. JEMS 1, 237-311 (1999)

3. F.H. Lin, T. Rivière: Complex Ginzburg-Landau Equations in High dimensions and minimal submanifolds II. In preparation

4. T. Rivière: Line vortices in the U(1)-Higgs model. C.O.C.V. 77-167 (1996) 